\title{
Predictores de satisfacción laboral en médicos y enfermeros ${ }^{1}$
}

\author{
Laura Paris \\ Universidad Católica Argentina \\ Alicia Omar \\ Consejo Nacional de Investigaciones Cientificas y Técnicas - Argentina
}

\begin{abstract}
Resumo
Os objetivos da presente investigação foram: (a) identificar estressores e estratégias de enfrentamento comumente empregadas por parte dos profissionais de saúde; (b) explorar as vinculações entre o estresse assistencial, o bem-estar e satisfação laboral; e (c) individualizar as variáveis explicativas da satisfação laboral assistencial. Foi realizada uma verificação empírica com uma amostra integrada por 196 profissionais argentinos (97 médicos e 99 enfermeiros). A amostra respondeu uma bateria desenvolvida para avaliar estressores, enfrentamento, bem-estar e satisfação laboral. Foram realizadas análises descritivas, correlacionais e de regressão múltipla. Os resultados obtidos indicaram que os estressores que mais afligem aos profissionais da saúde são a sobrecarga, a falta de apoio e a percepção de injustiça organizacional. Tanto a estratégia de resolução de problemas como a de distanciamento surgiram como potenciadoras de satisfação e bemestar. As variáveis que melhor explicaram a satisfação laboral foram a intenção de permanecer no cargo, a quantidade de horas de trabalho semanal e o apoio do grupo familiar. São apresentadas sugestões para futuras investigações na área.
\end{abstract}

Palavras-chave: estresse laboral; enfrentamento; satisfação laboral; bem-estar; médicos; enfermeiros

\begin{abstract}
Predictors of job satisfaction among physicians and nurses. The objectives of this research were: (a) to identify the major stressors and the coping strategies commonly used by health professionals; (b) to explore the links among health care stressors, subjective wellbeing and job satisfaction; and (c) to individualize the explanatory variables of care job satisfaction. Empirical verification was conducted with a sample of 196 Argentinean professionals (97 doctors and 99 nurses). The sample answered a battery developed for assessing stress, coping, wellbeing, and work satisfaction. Data were subjected to descriptive, correlation, and multiple regression analysis. The results indicated that work overload, lack of support, and perceptions of organizational injustice are the main health care stressors. The employment of problem-solving and distancing coping emerged as the best strategies for enhancing job satisfaction and wellbeing. The perception of familiar support, more working hours, and the intention to stay were the variables that proved to be the best predictors of work satisfaction. Suggestions are made for future research in the area.
\end{abstract}

Keywords: job stress; coping; job satisfaction; wellbeing; doctors; nurses

$\mathrm{T}$ Todas las profesiones y actividades laborales generan algún grado de estrés, aunque el trabajo vinculado con la atención de la salud se caracteriza por algunos estresores que son el resultado de una actitud de intensa dedicación al cuidado de la vida de los demás. En este sentido, el compromiso por la vida, las relaciones empático-afectivas con el enfermo y las características propias de las instituciones sanitarias, colocan a los trabajadores de la salud en una situación de riesgo permanente. Esta tensión cotidiana aumenta aún más, si los profesionales desempeñan sus funciones en instituciones sanitarias de países en vías de desarrollo. En el ámbito de las instituciones públicas, el recorte de las partidas presupuestarias, la insuficiencia de la infraestructura hospitalaria, la falta de medicamentos y la disminución de los salarios profesionales, configuran un clima laboral inestable y estresante (Camponovo Meier \& Morín Imbert, 2000). En el ámbito de las instituciones privadas, por su parte, las condiciones laborales se han ido deteriorando paulatinamente, configurando un ambiente de alta 
competitividad, que exige una dedicación no equivalente con las retribuciones económicas y sociales percibidas. La exposición constante a estas dificultades son responsables de los elevados niveles de estrés que padecen los profesionales de la salud (Zaldúa \& Lodieu, 2000).

Habitualmente, para enfrentar el estrés las personas recurren a respuestas cognitivas y comportamentales (proceso conocido como coping), que mediatizan las relaciones entre la amenaza percibida y la consiguiente adaptación somática y psicológica (Lazarus \& Folkman, 1984). La habilidad para manejar situaciones estresantes depende de los recursos de coping disponibles. Estos recursos desempeñan un rol crucial en la relación estrés-salud-enfermedad, constituyen características estables del individuo y se conocen como estrategias de coping o afrontamiento. Se han señalado asociaciones entre el empleo de estrategias de coping focalizadas en el problema y el bienestar, y entre las estrategias focalizadas en la emoción y la insatisfacción (McGowan, Gardner, \& Fletcher, 2006).

\section{Estrés laboral asistencial}

El estrés laboral ha sido definido como las reacciones individuales a las características del ambiente laboral percibidas como amenazantes por el trabajador (Martínez Selva, 2004). Surge de un desajuste (que puede ser crónico o agudo) entre las capacidades del individuo y las exigencias de su trabajo. En el ámbito asistencial, existe consenso entre los especialistas en clasificar las fuentes de estrés en dos grandes grupos: organizacionales y extraorganizacionales. Entre los principales estresores organizacionales se destacan la sobrecarga (Zacché Avellar, Iglesias, \& Fernández Valverde, 2007); los conflictos y la falta de apoyo de superiores y colegas (Salmond \& Ropis, 2005); la falta de recursos, tanto humanos como materiales (Grunfeld et al., 2005); la presión del tiempo (Ferraz Bianchi, 2004) y los bajos salarios, que generan una elevada percepción de inequidad (Montanholi, Tavares, \& Oliveira, 2006).

En cuanto a los estresores extraorganizacionales, se han identificado tanto variables individuales como contextuales. Entre las variables individuales, se han observado relaciones directas entre el aumento del estrés laboral, el nivel educativo y la cantidad de horas de trabajo (Lee \& Wang, 2002). Otras investigaciones han mostrado resultados controversiales entre el estrés laboral y variables sociodemográficas tales como la edad y el género (Muhonen \& Torkelson, 2004; Zambrano Plata, 2006), número de hijos (Collier, McCue, Markus, \& Smith, 2002) y el carácter público o privado de la institución (Enberg, Stenlund, Sundelin, \& Ohman, 2007). En relación con las variables psicológicas, se ha sugerido que la personalidad tipo D (Oginska-Bulik, 2006); el neuroticismo, la introversión (Huang, 2006) y el locus de control externo (Muhonen \& Torkelson, 2004) se asocian con mayores niveles de estrés. Además de tales indicadores individuales, en los últimos años se han identificado importantes asociaciones entre el estrés laboral y un conjunto de variables referentes al contexto social, tales como la incompatibilidad entre el trabajo y la vida familiar (Wainer, 2004), una pobre valoración social de la tarea (Guerrer \& Bianchi, 2008) y factores propios del ámbito laboral (Bazzoni, 2006; Elias \& Navarro, 2006).

\section{Estrés laboral asistencial y satisfacción con el tra- bajo}

La percepción de incapacidad para manejar las demandas laborales conduce a una disminución de la satisfacción con el trabajo. La satisfacción laboral es el resultado de una evaluación cognitiva mediante la qual el sujeto compara su realidad cotidiana con un estándar ideal construido a lo largo del tiempo. Si el resultado de tal comparación es positivo, el sujeto experimentará una sensación de bienestar y satisfacción (Ter Doest \& de Jonge, 2006). En caso contrario, vivenciará elevados niveles de estrés e insatisfacción (Cavalheiro, Moura Junior, \& Lopes, 2008; McGowan et al., 2008). Al respecto, Martín García, Luceño Moreno, Jaén Díaz y Rubio Valdehita (2007) han señalado que los trabajadores más estresados tienden a percibir su entorno laboral en forma más adversa y se evalúan como más insatisfechos con algunos aspectos de su trabajo. Asimismo, se han informado relaciones sinérgicas entre insatisfacción y estrés laboral (McClenahan, Giles, \& Mallett, 2007), desde el momento que a mayor insatisfacción se han registrado más síntomas de enfermedad.

Desde el punto de vista de la teoría transaccional del estrés (Lazarus \& Folkman, 1984), la satisfacción laboral se ha asociado con el empleo de determinadas de estrategias de coping. En el área asistencial, existe evidencia que los médicos con mayor satisfacción laboral son los que emplean coping más exitoso, tal como delegar tareas, organizar mejor la carga laboral, evaluar las posibilidades de manera más realista; mientras que los más insatisfechos recurren a la negación y al uso de alcohol y drogas (Nylenna, Gulbrandsen, Forde, \& Aasland, 2005). Investigaciones recientes subrayan el interjuego entre el estrés y la satisfacción laboral. En este sentido, por ejemplo, Karanikola, Papathanassoglou, Giannakopoulou y Koutroubas (2007) encuentran que los enfermeros con alta satisfacción laboral experimentan menores niveles de estrés; en tanto que López-Araújo, Osca Segovia y Peiró (2007) observan que los empleados que atribuyen gran importancia a su trabajo perciben menos estresores y experimentan mayor satisfacción laboral. En términos generales, los especialistas coinciden en señalar el impacto positivo y las consecuencias benéficas de la satisfacción con el trabajo tanto para el individuo como para la organización. Las evidencias indican que cuando la satisfacción laboral aumenta, disminuye el ausentismo y la rotación (Hayhurst, Saylor, \& Stuenkel, 2005), y se incrementan el compromiso y las actitudes positivas hacia el trabajo (Chiu \& Chen, 2005). Circunstancias que han llevado a señalar (Lee, Hwang, Kim, \& Daly, 2004) que la satisfacción laboral influiría positivamente en la satisfacción con la vida en general, aunque tales relaciones aún no están claramente establecidas.

Frente a este cuadro de situación, los propósitos de la presente investigación fueron los siguientes: (a) identificar las situaciones que en el ámbito sanitario argentino son percibidas como estresantes por médicos y enfermeros; (b) identificar las estrategias de afrontamiento más comúnmente empleadas por parte de los profesionales de la salud; (c) explorar las vinculaciones entre el estrés y su afrontamiento, con el bienestar y el grado de satisfacción laboral; y (d) conocer los principales 
predictores de satisfacción laboral entre los profesionales de la salud.

\section{Método}

\section{Muestra}

La verificación empírica se realizó sobre una muestra por disponibilidad integrada por 196 profesionales (97 médicos y 99 enfermeros) de instituciones sanitarias públicas y privadas de la ciudad de Rosario (Argentina). La elección de la muestra se basó en razones de factibilidad y accesibilidad. El 65\% eran mujeres; el promedio de edad de la muestra total fue de 38 años y el promedio de antigüedad laboral de 12 años. En cuanto al tipo de institución, el 32\% trabajaba en instituciones públicas (hospitales, centros de salud), el 40\% pertenecía a instituciones privadas (clínicas, atención domiciliaria) y el 27\% restante trabajaba en ambos tipos de instituciones.

\section{Procedimiento de recolección de datos}

El contacto con los profesionales se efectuó en los lugares de trabajo habituales y con previa solicitud de autorización a las autoridades sanitarias. La participación fue voluntaria y se tomaron todos los recaudos necesarios para garantizar el anonimato. Luego de hacerles conocer los objetivos del estudio, se invitó a los profesionales a formar parte de la investigación. En cada institución participante se instalaron urnas especialmente preparadas para que depositasen los protocolos una vez completados. Este mecanismo permitió que cada profesional contestara los cuestionarios en el lugar y momento en que le resultara más conveniente. Durante todo el proceso se tomaron en consideración todos los recaudos éticos vinculados con la investigación con seres humanos.

\section{Instrumentos}

La totalidad de la muestra respondió un cuadernillo integrado por los siguientes instrumentos:

\section{Escala de Estresores Asistenciales}

Los estresores asistenciales fueron explorados a través de la escala desarrollada por Paris (2007), integrada por 33 ítems, con formato tipo Likert de 4 puntos ( $0=$ nada; $3=$ mucho $)$. La escala explora cinco dimensiones o fuentes de estrés asistencial, a saber: falta de apoyo organizacional (ej.: “escasa disposición de pares y superiores para integrar equipos de trabajo”, $\alpha=$ 0,815); sobrecarga laboral (ej.: “excesivo número de pacientes a cargo", $\alpha=0,810$ ); dificultades interpersonales (ej.: "relaciones problemáticas con médicos o enfermeros”, $\alpha=0,774$ ); fuentes extrínsecas de insatisfacción (ej.: "bajos sueldos”, $\alpha=0,748$ ) y falta de justicia organizacional (ej.: "sensación de dar mucho en el trabajo y no ser recompensado”, $\alpha=0,723)$.

\section{Escala de Estrategias de Afrontamiento del Estrés Asistencial}

Las estrategias de afrontamiento fueron evaluadas a través de la escala desarrollada por Paris (2007), conformada por 24 ítems, con formato Likert de 3 puntos ( $0=$ nunca; $2=$ siempre $)$. El instrumento explora cuatro estrategias de afrontamiento: estrategias desadaptativas (ej.: "me evado del problema comiendo, fumando o bebiendo”, $\alpha=0,880$ ); estrategias de resolución de problemas (ej.: “planifico como resolver el problema”, $\alpha=0,757$ ); estrategias de resignación (ej.: “espero que las cosas cambien en algún momento”, $\alpha=0,781$ ) y estrategias de distanciamiento (ej.: "pienso en lo que haré en mi tiempo libre”, $\alpha=0,762$ ).

\section{Escala de Bienestar Subjetivo}

El bienestar subjetivo fue medido a través de la adaptación argentina (Paris, 2007) de la escala homónima desarrollada por Nacpal y Shell (1992). Para su validación se siguió el método divulgado por Moraes, Hasselmann y Reichenheim (2002). Vale decir que se efectuó la equivalencia conceptual y de ítems (a través de revisiones bibliográficas sobre el tema y debates con expertos); equivalencia semántica (a través de un proceso de traducción y retraducción); operacional (manteniendo las condiciones de administración en ambos contextos) y de medición (a través del cálculo de coeficientes de confiabilidad y validez). La versión validada quedó integrada por 40 ítems, con formato Likert de 3 puntos ( 1 = casi nunca; 3 = casi siempre). Si bien la escala original explora 11 factores a través de sus 40 ítems, la versión validada quedó integrada por 10 dimensiones habida cuenta que los análisis de componentes principales indicaron como la mejor solución la que integraba en un mismo factor las dos primeras dimensiones del instrumento original (bienestar vinculado a afectos positivos y correspondencia entre expectativas y logros). Las dimensiones medidas fueron las siguientes: correspondencia entre expectativas y logros (ej.: “¿piensa que ha logrado el estándar de vida que esperaba?”, $\alpha=$ 0,870); confianza en afrontar dificultades (ej.: “ ¿siente que puede manejar situaciones inesperadas?”, $\alpha=0,700$ ); trascendencia (ej.: “ ¿ha tenido alguna vez experiencias de intensa felicidad similar al éxtasis?”, $\alpha=0,766$ ); apoyo social (ej.: “¿siente que sus amigos y parientes lo ayudarían si estuviera necesitado?”, $\alpha=0,853$ ); relaciones con el grupo primario (ej.: “¿cómo se siente respecto a la relación con sus hijos?”, $\alpha=0,765)$; inadecuado manejo mental (ej.: “¿siente que es fácilmente irritable?”, $\alpha=0,843$ ); apoyo del grupo familiar (ej.: “¿considera que su familia lo ayuda a encontrar soluciones a la mayoría de sus problemas?”, $\alpha=$ 0,750); bienestar vinculado a afectos negativos (ej.: “¿siente que su vida es inservible?”, $\alpha=0,754)$; contactos sociales deficientes (ej.: “¿se preocupa a veces por no mantener una vinculación estrecha con alguien?”, $\alpha=0,759)$ y percepción de problemas de salud (ej.: “¿tiene problemas para dormir?”, $\alpha=0,767)$.

\section{Escala de Satisfacción Laboral y de Satisfacción} con la Vida en General

La satisfacción laboral y con la vida en general fue medida con la adaptación argentina (Paris, 2007) de la escala desarrollada por Shouksmith (1990). En este caso, también se siguió el mismo método de validación utilizado en la adaptación del instrumento anterior. La versión final reprodujo la original y quedó integrada por 12 ítems con 7 opciones de respuesta (1 = muy en desacuerdo; 7 = muy de acuerdo), agrupados en dos dimensiones: satisfacción laboral (ej.: “mi trabajo me da seguridad laboral”, $\alpha=0,807$ ) y satisfacción con la vida en general (ej.: “mi vida me permite 
desarrollar todas mis habilidades y potencialidades”, $\alpha=$ 0,806).

\section{Variables sociodemográficas}

El protocolo de recolección de datos se completó con un conjunto de preguntas orientadas a recabar información acerca de variables sociodemográficas tales como edad, sexo, estado civil, número de hijos, profesión, especialidad (en el caso de los médicos), ámbito laboral en que se desempeña, antigüedad, horas de trabajo semanal, intención de renunciar y desempeño en el ámbito de la docencia.

\section{Análisis de datos}

En primer lugar, y con el objetivo de detectar tanto las situaciones estresantes, como las estrategias de afrontamiento más comúnmente empleadas por los profesionales de la salud, se calcularon los índices descriptivos (medias y desviaciones típicas) correspondientes a cada una de estas variables. En segundo lugar, con el propósito de explorar las posibles vinculaciones entre estrés percibido, coping, bienestar subjetivo y satisfacción personal-laboral, se calcularon las correlaciones entre tales variables para la muestra total.

Finalmente, con el propósito de identificar los mejores predictores de la satisfacción laboral se calcularon un conjunto de análisis de regresión múltiple. Para la ejecución de dichos análisis se ingresaron, en el primer paso, las variables sociodemográficas con el propósito de controlar sus posibles efectos sobre la variable dependiente; en el segundo paso, se ingresaron los estresores asistenciales; en el tercer paso, las estrategias de afrontamiento del estrés; en el cuarto paso la percepción de satisfacción con la vida y, por último, en el quinto paso, las dimensiones del bienestar subjetivo. Tales variables desempeñaron el rol de independientes, en tanto que la satisfacción laboral adoptó el rol de variable dependiente.

El orden de entrada de las variables se fundamentó tanto en su relevancia teórica (teoría transaccional del estrés de Lazarus \& Folkman, 1984), como en la evidencia empírica publicada. En este sentido, las variables sociodemográficas se ingresaron en el primer paso, atendiendo a los hallazgos informados por Lepnurm, Dobson, Backman y Keegan (2006); Rondeau y Francescutti (2005) y Wilson (2006). Los estresores asistenciales se ingresaron en el segundo paso, en base a las observaciones efectuadas por Hyrkäs (2005), Laschinger y Finegan (2005) y Ter Doest y de Jonge (2006). En el tercer paso se ingresaron las estrategias de afrontamiento, dado que su asociación con la satisfacción laboral ha recibido menor apoyo empírico (Thomas, 2005). La satisfacción con la vida fue relegada al cuarto lugar, porque la evidencia empírica de su rol predictivo es un tanto contradictoria (Lee et al., 2004; Martínez Selva, 2004). Las dimensiones de bienestar subjetivo se ingresaron en el último paso ya que su asociación con la satisfacción laboral no se han documentado a través de las múltiples facetas del constructo que se han tomado en cuenta en el presente estudio, sino que sólo se han informado vinculaciones acotadas a ciertos aspectos del trabajo, tales como las relaciones armoniosas con pares y superiores (Gilbreath \& Benson, 2004), la realización personal (Cotton \& Hart, 2003) y el clima organizacional (Shanafelt et al., 2005).

\section{Resultados}

En la Tabla 1 se presentan los índices descriptivos (medias y desviaciones típicas) correspondientes a cada una de las situaciones percibidas como estresantes y a las estrategias de afrontamiento más utilizadas por los profesionales de la salud en su conjunto $(N=196)$. Como se observa, el estresor que

Tabla 1

Medias y desvíos típicos correspondientes a los estresores asistenciales y las estrategias de afrontamiento (Muestra total)

\begin{tabular}{llcc}
\hline \multicolumn{1}{c}{ Variables } & \multicolumn{1}{c}{ Dimensiones* } & $X$ & $s$ \\
\hline Estresores Asistenciales & Sobrecarga & 1,50 & 0,72 \\
& Falta de apoyo organizacional & 1,35 & 0,73 \\
& Falta de justicia organizacional & 1,35 & 0,70 \\
& Fuentes extrínsecas & 1,23 & 0,63 \\
& Dificultades interpersonales & 0,96 & 0,61 \\
\hline \multirow{2}{*}{ Estrategias de afrontamiento } & Resolución de problemas & 1,49 & 0,35 \\
& Distanciamiento & 1,17 & 0,35 \\
& Resignación & 0,75 & 0,33 \\
& Estrategias desadaptativas & 0,47 & 0,30 \\
\hline
\end{tabular}

* las subdimensiones están ordenadas en función del valor de la media 
más afecta a médicos y enfermeros es la sobrecarga por exceso de pacientes, tareas y demandas. Seguido por las percepciones de falta de apoyo y de justicia organizacional que conllevan la sensación de inequidad inter e intrapersonal, imposibilidad de contar con la ayuda de pares y superiores, falta de autonomía para tomar decisiones, pobre devolución de desempeño, entre otros.

La Tabla 1 también muestra que las estrategias de coping empleadas con mayor frecuencia por los profesionales de la salud son la de resolución de problemas y el distanciamiento de la fuente de estrés. La estrategia de resolución de problemas incluye tanto los esfuerzos por solucionar la situación estresante como por buscar apoyo social. La estrategia de distanciamiento se refiere a los esfuerzos cognitivos por alejarse de la situación estresante, planificando, por ejemplo, actividades placenteras para el tiempo libre.

La matriz de correlaciones (Tabla 2) permite observar las vinculaciones entre la totalidad de las variables en estudio. Como se advierte, los mayores niveles de estrés asistencial se asocian positivamente con estrategias desadaptativas ( $r=$ $0,17 ; p<0,05)$ y negativamente con estrategias de resolución de problemas $(r=-0,16 ; p<0,05)$. Estas asociaciones están indicando que a mayor estrés, menor coping de acción directa y mayor predominio de estrategias ineficaces tales como evadirse a través del alcohol o del cigarrillo, descargar la tensión en otros, somatizar, responder cínicamente o automedicarse. A su vez, a mayor estrés, se observan bajos niveles de satisfacción laboral $(r=-0,57 ; p<0,01)$, de satisfacción personal $(r=-0,41 ; p<$ $0,01)$ y de bienestar $(r=-0,27 ; p<0,01)$.

En cuanto a las interrelaciones entre coping, satisfacción laboral y bienestar, se observan dos tendencias diferentes. Por un lado, el empleo de estrategias desadaptativas $(r=-0,31 ; p<0,01)$ y de resignación $(r=-0,24 ; p<0,01)$ se vinculan negativamente con la satisfacción con la vida. Mientras que por otro lado, el empleo de estrategias de resolución de problemas ( $r=0,27$; $p<0,01)$ y de distanciamiento $(r=0,27 ; p<0,01)$ se asocian positivamente con el bienestar.

Finalmente, a efectos de identificar los predictores de satisfacción laboral se calcularon un conjunto de análisis de regresión múltiple, tanto para la muestra total (Tabla 3) como para médicos (Tabla 4) y enfermeros (Tabla 5) por separado.

Como se desprende de la Tabla 3, para los profesionales de la salud en su conjunto, sin discriminar entre médicos y enfermeros, las variables sociodemográficas referidas a cantidad de horas de trabajo diario e intención de mantener el empleo (ingresadas en el primer paso), contribuyen significativamente a la ecuación de regresión, explicando el 5,6\% $\left(\Delta R^{2}=0,056\right)$ de la varianza de la satisfacción laboral. En el segundo paso, los estresores referidos a fuentes extrínsecas de insatisfacción, falta de apoyo organizacional y dificultades interpersonales, contribuyen con el $39 \%\left(\Delta R^{2}=0,446-0,056=0,390\right)$, siendo el cambio en $R^{2}(0,500-0,124=0,376)$ significativo al nivel de 0,001 . Cuando se incorporaron al modelo las estrategias de afrontamiento, explicaron un 3,6\% adicional $(0,482-0,446=$ $0,036)$, no alcanzando el cambio en $R^{2}(0,543-0,500=0,043)$ significación a los niveles convencionales. En el cuarto paso, la satisfacción con la vida contribuyó con un 3,3\% (0,515 $-0,482=0,033)$ a la explicación de la satisfacción laboral, no obstante, el cambio en $R^{2}(0,575-0,543=0,032)$ no alcanzó significación estadística. Finalmente, al incorporar las dimensiones del bienestar subjetivo, éstas contribuyeron con un 3\% suplementario a la explicación de la variable dependiente $(0,545-0,515=0,030)$, aunque el cambio en $R^{2}(0,625-0,575$ $=0,050)$ tampoco alcanzó significación estadística. En total, las variables predictoras explicaron un 54,5\% (5,6 + 39,0 + 3,6+ $3,3+3,0$ ) de la satisfacción laboral.

La Tabla 4 muestra el comportamiento de las variables exploradas en la explicación de la satisfacción laboral de los médicos $(N=97)$.

Como se observa, de las variables sociodemográficas ingresadas en el primer paso, sólo la cantidad de horas de trabajo contribuyó significativamente a la ecuación de regresión, explicando el $7 \%\left(\Delta R^{2}=0,070\right)$ de la varianza de la satisfacción laboral. En el paso 2, los estresores referidos a fuentes extrínsecas de insatisfacción y falta de apoyo organizacional contribuyeron con el $41,6 \%\left(\Delta R^{2}=0,486-0,070=0,416\right)$, siendo el cambio en $R^{2}(0,588-0,206=0,382)$ significativo al nivel de 0,001 . Cuando se incorporaron al modelo las estrategias de afrontamiento, solo agregaron un $1,2 \%(0,498-0,486=0,012)$, no alcanzando el cambio en $R^{2}(0,619-0,588=0,031)$ significación a los niveles esperables. En el cuarto paso, la satisfacción con la vida contribuyó con un 4,4\% $(0,542-0,498=0,044)$, careciendo el cambio en $R^{2}(0,657-0,619=0,038)$ de significación estadística. Finalmente, al incorporar las dimensiones del bienestar subjetivo, éstas contribuyeron con un 6,8 \% adicional $(0,610-0,542$ $=0,068)$, siendo el cambio en $R^{2}(0,748-0,657=0,091)$ significativo al nivel de 0.05 . Por lo que en total las variables predictoras explicaron un $61 \%(7,0+41,6+1,2+4,4+6,8)$ de la satisfacción laboral de los médicos.

La Tabla 5 muestra el comportamiento de la totalidad de las variables exploradas en la explicación de la satisfacción laboral de los enfermeros $(N=99)$.

En el primer paso, la única variable sociodemográfica que contribuyó significativamente a la ecuación de regresión fue la intención de permanecer en el puesto, explicando el 10,6\% de la varianza de la satisfacción laboral entre los enfermeros. En el paso dos, los estresores vinculados con la falta de apoyo organizacional y con las fuentes extrínsecas de insatisfacción contribuyeron con un 30,7\% adicional $(0,413-0,106=0,307)$, siendo el cambio en $R^{2}(0,527-0,233=0,294)$ significativo al nivel de 0,001 . Cuando se incorporaron al modelo las estrategias de afrontamiento, agregaron un 9,6\% $(0,509-0,413=0,096)$, siendo el cambio en $R^{2}(0,624-0,527=0,097)$ significativo al nivel de 0,05 . En el cuarto paso, la satisfacción con la vida no efectuó aportes significativos a la explicación de la variable dependiente. Finalmente, al incorporar las dimensiones del bienestar subjetivo, éstas contribuyeron con un 4,3\% (0,559 $0,516=0,043)$ no alcanzando el cambio en $R^{2}(0,712-0,635=$ $0,077)$ significación estadística. En consecuencia, las variables predictoras explicaron el 55,2\% (10,6 + 30,7 + 9,6 + 4,3) de la satisfacción laboral de los enfermeros. 
Tabla 2

Coeficientes de correlaciones entre estresores, estrategias de afrontamiento, satisfacción laboral-personal y bienestar en la muestra total (médicos y enfermeros)

\begin{tabular}{lcccccccc}
\hline & 1. & 2. & 3. & 4. & 5. & 6. & 7. & 8. \\
\hline 1. Estrés total & - & $0,17^{*}$ & $-0,16^{*}$ & 0,10 & $-0,10$ & $-0,57^{* *}$ & $-0,41^{* *}$ & $-0,27^{* *}$ \\
2. Estrategias desadaptativas & & - & $-0,18^{*}$ & 0,05 & $-0,08$ & $-0,15^{*}$ & $-0,31^{* *}$ & $-0,41^{* *}$ \\
3. Resolución de problemas & & & - & $-0,11$ & $0,25^{* *}$ & $0,27^{* *}$ & $0,38^{* *}$ & $0,27^{* *}$ \\
4. Resignación & & & & - & 0,01 & $-0,01$ & $-0,24^{* *}$ & $-0,07$ \\
5. Distanciamiento & & & & & - & 0,11 & $0,23^{* *}$ & $0,27^{* *}$ \\
6. Satisfacción laboral & & & & & & - & $0,46^{* *}$ & $0,30^{* *}$ \\
7. Satisfacción con la vida & & & & & & & - & $0,51^{* *}$ \\
8. Bienestar subjetivo & & & & & & & & - \\
\hline
\end{tabular}

$* p<0,05 ; * * p<0,01$

Tabla 3

Predictores de satisfacción laboral en la muestra total

\begin{tabular}{|c|c|c|}
\hline \multirow{2}{*}{ Variables predictoras } & \multicolumn{2}{|c|}{ Variable dependiente: Satisfacción laboral } \\
\hline & $\beta$ estandarizada & $F$ \\
\hline \multicolumn{3}{|c|}{ Primer paso (variables sociodemográficas) } \\
\hline Intención de permanecer & $0,265 * * *$ & \\
\hline Horas & $0,213^{* *}$ & \\
\hline $\begin{aligned} R^{2} & =0,124 \\
\Delta R^{2} & =0,056\end{aligned}$ & & $F(14,181)=1,825^{*}$ \\
\hline \multicolumn{3}{|c|}{ Segundo paso (estresores) } \\
\hline Fuentes extrínsecas de insatisfacción & $-0,400 * * *$ & \\
\hline Falta de apoyo organizacional & $-0,301 * * *$ & \\
\hline Dificultades interpersonales & $-0,185^{* *}$ & \\
\hline $\begin{aligned} R^{2} & =0,500 \\
\Delta R^{2} & =0,446\end{aligned}$ & & $F(19,176)=9,264 * * *$ \\
\hline \multicolumn{3}{|c|}{ Tercer paso (afrontamiento) } \\
\hline $\begin{array}{l}\text { Resolución de problemas } \\
\qquad \begin{aligned} R^{2} & =0,543 \\
\Delta R^{2} & =0,482\end{aligned}\end{array}$ & $0,176^{*}$ & $F(23,172)=8,888 * * *$ \\
\hline \multicolumn{3}{|c|}{ Cuarto paso (satisfacción con la vida) } \\
\hline Satisfacción con la vida & $0,234 * *$ & \\
\hline $\begin{aligned} R^{2} & =0,575 \\
\Delta R^{2} & =0,515\end{aligned}$ & & $F(24,171)=9,641^{* * *}$ \\
\hline \multicolumn{3}{|c|}{ Quinto paso (bienestar subjetivo) } \\
\hline Correspondencia expectativas y logros & $0,216^{* *}$ & \\
\hline Manejo mental & $0,204 * *$ & \\
\hline Apoyo del grupo familiar & $0,140 *$ & \\
\hline $\begin{aligned} R^{2} & =0,625 \\
\Delta R^{2} & =0,545\end{aligned}$ & & $F(34,161)=7,880 * * *$ \\
\hline
\end{tabular}

$* p<0,05 ; * * p<0,01 ; * * * p<0,001$ 
Tabla 4

Predictores de satisfacción laboral en médicos

\begin{tabular}{|c|c|c|}
\hline \multirow{2}{*}{ Variables predictoras } & \multicolumn{2}{|c|}{ Variable dependiente: Satisfacción laboral } \\
\hline & $\beta$ estandarizada & $F$ \\
\hline \multicolumn{3}{|c|}{ Primer paso (variables sociodemográficas) } \\
\hline Horas & $0,404 * * *$ & \\
\hline $\begin{aligned} R^{2} & =0,206 \\
\Delta R^{2} & =0,070\end{aligned}$ & & $F(14,82)=1,519$ \\
\hline \multicolumn{3}{|c|}{ Segundo paso (estresores) } \\
\hline Fuentes extrínsecas de insatisfacción & $-0,349 * * *$ & \\
\hline Falta de apoyo organizacional & $-0,329 * * *$ & \\
\hline $\begin{aligned} R^{2} & =0,588 \\
\Delta R^{2} & =0,486\end{aligned}$ & & $F(19,77)=5,777^{* * *}$ \\
\hline \multicolumn{3}{|c|}{ Tercer paso (afrontamiento) } \\
\hline \multicolumn{3}{|l|}{ No ingresan variables al modelo } \\
\hline $\begin{aligned} R^{2} & =0,619 \\
\Delta R^{2} & =0,498\end{aligned}$ & & $F(23,73)=5,148^{* * *}$ \\
\hline \multicolumn{3}{|c|}{ Cuarto paso (satisfacción con la vida) } \\
\hline Satisfacción con la vida & $0,257^{* *}$ & \\
\hline $\begin{aligned} R^{2} & =0,657 \\
\Delta R^{2} & =0,542\end{aligned}$ & & $F(24,72)=5,741^{* * *}$ \\
\hline \multicolumn{3}{|c|}{ Quinto paso (bienestar subjetivo) } \\
\hline Correspondencia expectativas y logros & $0,326 * * *$ & \\
\hline Apoyo del grupo familiar & $0,201^{* *}$ & \\
\hline $\begin{aligned} R^{2} & =0,748 \\
\Delta R^{2} & =0,610\end{aligned}$ & & $F(34,62)=5,419^{* * *}$ \\
\hline
\end{tabular}

** $p<0,01$; *** $p<0,001$ 
Tabla 5

Predictores de satisfacción laboral en enfermeros

\begin{tabular}{|c|c|c|}
\hline \multirow{2}{*}{ Variables predictoras } & \multicolumn{2}{|c|}{ Variable dependiente: Satisfacción laboral } \\
\hline & $\beta$ estandarizada & $F$ \\
\hline \multicolumn{3}{|c|}{ Primer paso (variables sociodemográficas) } \\
\hline Intención de permanecer & $0,368^{* * *}$ & \\
\hline $\begin{aligned} R^{2} & =0,233 \\
\Delta R^{2} & =0,106\end{aligned}$ & & $F(14,84)=1,827^{*}$ \\
\hline \multicolumn{3}{|c|}{ Segundo paso (estresores) } \\
\hline Fuentes extrínsecas de insatisfacción & $-0,298 * * *$ & \\
\hline Falta de apoyo organizacional & $-0,322 * * *$ & \\
\hline $\begin{aligned} R^{2} & =0,527 \\
\Delta R^{2} & =0,413\end{aligned}$ & & $F(19,79)=4,631^{* * *}$ \\
\hline \multicolumn{3}{|c|}{ Tercer paso (afrontamiento) } \\
\hline Resolución de problemas & $0,244^{* *}$ & \\
\hline Afrontamiento desadaptativo & $-0,170 *$ & \\
\hline $\begin{aligned} R^{2} & =0,624 \\
\Delta R^{2} & =0,509\end{aligned}$ & & $F(23,75)=5,409 * * *$ \\
\hline \multicolumn{3}{|c|}{ Cuarto paso (satisfacción con la vida) } \\
\hline $\begin{array}{l}\text { No ingresan variables al modelo } \\
\qquad R^{2}=0,635 \\
\Delta R^{2}=0,516\end{array}$ & & $F(24,74)=5,355^{* * *}$ \\
\hline Quinto paso (bienestar & ivo) & \\
\hline Manejo mental & $0,325^{* * *}$ & \\
\hline Percepción de salud & $0,268 * * *$ & $F(34,64)=4,653^{* * *}$ \\
\hline
\end{tabular}

${ }^{*} p<0,05 ; * * p<0,01 ; * * * p<0,001$

\section{Consideraciones finales}

La presente investigación se ha desarrollado dentro del marco teórico provisto por la teoría transaccional del estrés de Lazarus y Folkman (1984). Desde esta perspectiva, la experiencia del estrés es una construcción de naturaleza predominantemente subjetiva desde el momento que los individuos movilizan tanto factores personales como situacionales para evaluar el potencial perjudicial de los eventos. Cuando el sujeto siente que no cuenta con los recursos o estrategias suficientes para afrontar las demandas que percibe como estresantes, experimenta diferentes niveles de estrés que pueden llegar a vulnerar su equilibrio y bienestar psicológicos. Si, además, el estrés es vivenciado en el ámbito laboral, puede repercutir sobre la calidad de su trabajo, el nivel de compromiso organizacional y su grado de satisfacción laboral-personal.

Con base en tales consideraciones, uno de los objetivos del presente estudio estuvo orientado a la identificación de los estresores asistenciales y de las estrategias para afrontarlos entre los médicos y enfermeros que desempeñan su trabajo en el ámbito de uno de los mayores conglomerados urbanos argentinos (ciudad de Rosario). En cuanto a los estresores asistenciales, los resultados indicaron que la sobrecarga laboral, la falta de apoyo y la percepción de injusticia organizacional, son, en ese orden, los estresores que más los agobian. Se trataría de un conjunto de estresores que se retroalimentan permanentemente constituyendo un círculo vicioso, desde el momento que la sobrecarga (caracterizada por una sensación de agotamiento por exceso de demandas y tareas), unida a la falta de medios y recursos, se agrava cuando los profesionales perciben falta de apoyo por parte de pares y superiores y/o inequidad e injusticia organizacional. Estos resultados están en consonancia con los informados por otros investigadores, tanto argentinos (Zaldúa \& Lodieu, 2000) como extranjeros (Lambert, Lambert, Petrini, \& Zhang, 2007; Nascimento Ramalho \& Fantini NogueiraMartins, 2007). 
Con respecto a las estrategias de afrontamiento, los resultados mostraron que los profesionales de la salud tienden al empleo de estrategias de resolución de problemas (que incluyen la búsqueda de apoyo social) y de distanciamiento (que incluyen cogniciones vinculadas con el ocio y el empleo del tiempo libre). Las diferentes facetas de la búsqueda de apoyo social (tales como hablar con colegas, integrar equipos de trabajo o tratar de llegar a acuerdos) han sido asociadas con mayores niveles de bienestar (Archibald, 2006; Luceño Moreno, Martín García, Jaén Díaz, \& Díaz Ramiro, 2006); en tanto que la estrategia de distanciamiento ha sido identificada como promotora de satisfacción (Caldwell, 2005; Iwasaki, 2003) y su empleo ha sido observado entre médicos y enfermeros de las más diferentes culturas (Lambert et al., 2007; Taylor, Pallant, Crook, \& Cameron, 2004).

La evidencia reunida también ha permitido corroborar el interjuego entre el estrés percibido, su afrontamiento, el bienestar y la satisfacción personal-laboral. En este sentido, se ha observado que los profesionales que emplean estrategias de afrontamiento más desadaptativas, perciben más estresores y experimentan menos satisfacción y menos bienestar. Esta relación ha sido documentada en similares grupos ocupacionales (Arafa, Nazel, Ibrahim, \& Attia, 2003; Ferrareze, Ferreira, \& Carvalho, 2006), así como entre empleados de otros rubros (Lu, While, \& Barriball, 2007; Martín García et al., 2007) e, incluso, entre soldados profesionales (López-Araújo et al., 2007). Tal hallazgo también está en sintonía con los comunicados por Mann Layne, Hohenshil y Singh (2004) quienes detectaron que al aumentar el estrés disminuyen los recursos de afrontamiento de acción directa y aumentan los síntomas de malestar; y con los de Tabak y Koprak (2007) quienes advirtieron que la ineficacia del coping de evitación se asocia con mayores niveles de estrés e insatisfacción. Paralelamente, se ha registrado la tendencia inversa, es decir, a menor estrés, mayores niveles de satisfacción y bienestar, sobre todo entre los que emplean estrategias de resolución de problemas y de distanciamiento. Tales evidencias estarían indicando que estas dos estrategias de coping actuarían como potenciadoras de bienestar y satisfacción, lo que coincide con lo señalado por Dolbier, Smith y Steinhardt (2007) y por Tugade, Fredrickson y Barret (2004).

Los resultados obtenidos, a su vez, han permitido identificar predictores de satisfacción laboral entre los profesionales de la salud. La intención de permanecer en el puesto de trabajo, el afrontamiento de resolución de problemas, la satisfacción con la vida y algunas dimensiones del bienestar (tales como la correspondencia entre expectativas y logros, el adecuado manejo mental y el apoyo percibido por parte del grupo familiar), emergieron como los mejores predictores de la satisfacción laboral asistencial. En tanto que los aspectos extrínsecos al trabajo, la falta de apoyo organizacional y las dificultades interpersonales, surgieron como los predictores más fuertes de la insatisfacción laboral. Similares resultados han sido reseñados en la bibliografía especializada (Archibald, 2006; Lepnurm et al., 2006; McGillis Hall \& Doran, 2007), lo que muestra que más allá de las diferencias culturales, hay aspectos de la vida laboral que trascienden los contextos sociales y políticos, locales o regionales. Finalmente, al analizar los determinantes de la satisfacción laboral en función de la profesión, se pudo observar que la percepción de apoyo organizacional y familiar, la coherencia entre expectativas y logros, y las mayores posibilidades de ascenso y promociones, son los principales predictores de satisfacción entre los médicos. En tanto que los enfermeros más satisfechos con su trabajo son aquellos que tienen intenciones de permanecer en su puesto de trabajo, perciben apoyo laboral, resuelven los problemas de manera activa y cooperativa, tienen un adecuado manejo mental y gozan de buena salud.

\section{Fortalezas, limitaciones y sugerencias para futuros estudios}

Como toda investigación empírica, el presente estudio se caracteriza por algunas debilidades y fortalezas. Entre las debilidades hay que remarcar la composición de la muestra en estudio, ya que por haber estado integrada por disponibilidad impediría la generalización de los resultados a toda la población de profesionales de la salud argentinos. No obstante y, en sintonía con lo puntualizado por un gran número de investigadores en el área (Ferraz Bianchi, 2004; Salmond \& Ropis, 2005; Skytt, Ljunggren \& Carlsson, 2007), como en la composición de la muestra se incluyeron representantes de las más diversas instituciones asistenciales del medio, los resultados obtenidos podrían considerarse un fiel reflejo de la situación asistencial local. Otra limitación del estudio podría estar referida al carácter auto descriptivo de la mayoría de los instrumentos empleados para la recolección de los datos. Aspecto que podría haber generado tanto sesgos derivados de la varianza del método común como una mayor tendencia a la deseabilidad social. En este sentido, los participantes pueden haber ofrecido respuestas movilizadas por el deseo de presentar una imagen mejorada de sí mismos, ocultando o dejando de reconocer algunos síntomas social y/o laboralmente mal vistos. Sin embargo, previendo tales contingencias, se tomaron todos los recaudos necesarios para garantizar el carácter anónimo no sólo del protocolo sino también del proceso de devolución de los formularios completados, evitando que jefes y supervisores pudieran tener acceso a la información suministrada por sus subalternos. Este último aspecto se vincula a lo que podría constituir una tercera limitación de este estudio, ya que el proceso de recolección de datos demandó casi seis meses debido a las dilaciones y retrasos en la devolución de los cuestionarios.

Entre las fortalezas, hay que destacar el empleo de instrumentos de naturaleza émica que contribuyen a aumentar la validez y confiabilidad de los hallazgos (Cea D'Ancona, 1998). Los dos instrumentos desarrollados para esta ocasión (Escalas de Estresores Asistenciales y de Estrategias de Afrontamiento del Estrés Asistencial), basados en las características idiosincráticas de la población objetivo, posiblemente han permitido captar más apropiadamente el significado connotativo de los constructos investigados y la intensidad y dirección de sus interrelaciones (Naswall, Sverke, \& Hellgren, 2005; Salmond \& Ropis, 2005). Por sus características y propiedades psicométricas, se trata de instrumentos que podrían reemplazar a los utilizados hasta el momento, los que por provenir de contextos laborales foráneos quizá no llegaban a captar todos los matices de la problemática local. Se destaca, además, que esta investigación constituye una 
contribución genuina al conocimiento de la sinergia estresores asistenciales-afrontamiento del estrés ya que, en sintonía con las sugerencias de los especialistas internacionales sobre el tema, no sólo se ha incluido el análisis de las circunstancias y características personales (McNeely, 2005), sino que también se ha profundizado en el rol del apoyo social (Ito, Eisen, Sederer, Yamada, \& Tachimori, 2001) y en las repercusiones del afrontamiento focalizado en la emoción (Folkman \& Moskowitz, 2004).

A la luz de los resultados obtenidos, y como orientación para futuras investigaciones, se sugiere profundizar el estudio de las percepciones de justicia organizacional en el seno de las instituciones asistenciales. Se recomienda explorar no sólo la justicia vinculada con la distribución de los beneficios y con la implementación de los procedimientos, sino también las percepciones de justicia interaccional (Omar, 2006), habida cuenta la importancia atribuída tanto por médicos como enfermeros a la calidad de las relaciones interpersonales en su trabajo cotidiano. Asimismo, en futuros estudios se podría analizar el impacto de los comportamientos extra papel (Chiu \& Chen, 2005) sobre el bienestar y la satisfacción laboral asistencial. Sobre todo porque el trabajo propio de algunos departamentos hospitalarios (urgencias, cirugías y similares) se asienta en la ayuda y la colaboración permanente entre los profesionales. Es posible que si perciben justicia, se involucren con más frecuencia en la ejecución de comportamientos extra papel positivos (tales como altruísmo, cortesía, lealtad y similares). Como también es posible que si perciben injusticias, protagonicen comportamientos extra papel negativos (tales como acoso, revancha, represalia, y similares), los que, a la postre, pueden repercutir sobre el cuidado y atención de los propios pacientes.

Como corolario del trabajo realizado, se sugiere poner en marcha programas de intervención para incrementar la satisfacción laboral en los servicios de salud. Tales programas podrían consistir tanto en el entrenamiento en estrategias para afrontar eficazmente el estrés, como en la implementación regular de espacios grupales de diálogo que favorezcan el desarrollo del apoyo interpersonal. Estas instancias de interacción podrían cumplir un rol preventivo, anticipando la aparición de trastornos sociopsicológicos que erosionan el bienestar y la satisfacción personal, al tiempo que contribuirían a crear una atmósfera laboral atravesada por el compromiso de mejorar continuamente.

\section{Referencias}

Arafa, M., Nazel, M., Ibrahim, N., \& Attia, A. (2003). Predictors of psychological well-being of nurses in Alexandria, Egipt. International Journal of Nursing Practice, 9, 313-320.

Archibald, C. (2006). Job satisfaction among neonatal nurses. Pediatric Nursing, 32, 176-179.

Bazzoni, C. (2006, Junio 11). El sistema de salud rosarino tiene sólo una enfermera por cada dos médicos. Diario La Capital on line.

Caldwell, L. (2005). Leisure and health: why is leisure therapeutic? British Journal of Guidance \& Counselling, 33, 7-26.
Camponovo Meier, O., \& Morín Imbert, P. (2000). Síndrome de burnout en el personal de salud de un hospital público de la ciudad de Rosario. Investigación en Salud, 3(1-2), 73-93.

Cavalheiro, A. M., Moura Junior, D. F., \& Lopes, A. C. (2008). Stress in nurses working in intensive care units. Revista Latino-Americana de Enfermagem, 16(1), 29-35.

Cea D’Ancona, M. A. (1998). Metodología cuantitativa. Estrategias y técnicas de investigación social. Madrid: Síntesis.

Chiu, S., \& Chen, H. (2005). Relationship between job characteristics and organizational citizenship behavior: the mediational role of job satisfaction. Social Behavior and Personality, 33, 523-540.

Collier, V., McCue, J., Markus, A., \& Smith, L. (2002). Stress in medical residency: status quo after a decade of reform? Annals of Internal Medicine, 136, 384-390.

Cotton, P., \& Hart, P. (2003). Occupational wellbeing and performance: a review of organisational health research. Australian Psychologist, 38, 118-127.

Dolbier, C., Smith, S., \& Steinhardt, M. (2007). Relationships of protective factors to stress and symptoms of illness. American Journal of Health Behavior, 31, 423-433.

Elias, M., \& Navarro, V. (2006). A relação entre o trabalho, a saúde e as condições de vida: negatividade e positividade no trabalho das profissionais de enfermagem de um hospital escola. Revista Latino-Americana de Enfermagem, 14(4), 517-525.

Enberg, B., Stenlund, H., Sundelin, G., \& Ohman, A. (2007). Work satisfaction, career preferences and unpaid household work among recently graduated health-care professionals - a gender perspective. Scandinavian Journal of Caring Science, 21, 169-177.

Ferrareze, M. V. G., Ferreira, V., \& Carvalho, A. M. P. (2006). Percepção do estresse entre enfermeiros que atuam em terapia intensiva. Acta Paulista de Enfermagen ,19(3), 310-315.

Ferraz Bianchi, E. (2004). Stress and coping among cardiovascular nurses: a survey in Brazil. Issues in Mental Health Nursing, 25, 737-745.

Folkman, S., \& Moskowitz, J. (2004). Coping: pitfalls and promise. Annual Review of Psychology, 55, 745-774.

Gilbreath, B., \& Benson, P. (2004). The contribution of supervisor behavior to employee psychological well-being. Work \& Stress, 18, 255-266.

Grunfeld, E., Zitzelsberger, L., Coristine, M., Whelan, R., Aspelund, F., \& Evans, W. (2005). Job stress and job satisfaction of cancer care workers. Psychooncology, 14, 61-69.

Guerrer, F. J. L., \& Bianchi, E. R. F. (2008). Caracterização do estresse nos enfermeiros de unidades de terapia intensiva. Revista da Escola de Enfermagen da USP, 42(2), 355-362.

Hayhurst, A., Saylor, C., \& Stuenkel, D. (2005). Work environmental factors and retention of nurses. Journal of Nursing Care Quality, 20, 283-288.

Huang, H. (2006). Personality traits reflect employee job attitudes in the workplace. The Consortium Journal of Hospitality and Tourism, 10, 31-43.

Hyrkäs, K. (2005). Clinical supervision, burnout, and job satisfaction among mental health and psychiatric nurses in Finland. Issues in Mental Health Nursing, 26, 531-556.

Ito, H., Eisen, S., Sederer, L., Yamada, O. \& Tachimori, H. (2001). Factors affecting psychiatric nurses' intention to leave their current job. Psychiatric Services, 52, 232-234.

Iwasaki, Y. (2003). Examining rival models of leisure coping mechanisms. Leisure Sciences, 25, 183-203.

Karanikola, M., Papathanassoglou, E., Giannakopoulou, M., \& Koutroubas, A. (2007). Pilot exploration of the association between self-esteem and professional satisfaction in Hellenic hospital nurses. Journal of Nursing Management, 15, 78-90.

Lambert, V., Lambert, C., Petrini, M., \& Zhang, Y. (2007). Predictors of physical and mental health in hospital nurses within the People's Republic of China. International Nursing Review, 54, 85-91.

Laschinger, H., \& Finegan, J. (2005). Using empowerment to build trust and 
respect in the workplace: a strategy for addressing the nursing shortage. Nursing Economic, 23, 6-13.

Lazarus, R., \& Folkman, S. (1984). Stress, appraisal and coping. Nueva York: Springer.

Lee, H., Hwang, S., Kim, J., \& Daly, B. (2004). Predictors of life satisfaction of Korean nurses. Journal of Advanced Nursing, 48, 632-641.

Lee, I., \& Wang, H. (2002). Perceived occupational stress and related factors in public health nurses. Journal of Nursing Research, 10, 253-259.

Lepnurm, R., Dobson, R., Backman, A., \& Keegan, D. (2006). Factors explaining career satisfaction among psychiatrists and surgeons in Canada. Canadian Journal of Psychiatry, 51, 243-255.

López-Araújo, B., Osca Segovia, A., \& Peiró, J. (2007). El papel modulador de la implicación con el trabajo en la relación entre el estrés y la satisfacción laboral. Psicothema, 19, 81-87.

Lu, H., While, A., \& Barriball, K. (2007). A model of job satisfaction of nurses: a reflection of nurses' working lives in Mainland China. Journal of Advanced Nursing, 58, 468-479.

Luceño Moreno, L., Martín García, J., Jaén Díaz, M., \& Díaz Ramiro, E. (2006). Psycho-social risks and trait anxiety as predictors of stress and job satisfaction. Ansiedad y Estrés, 12, 89-97.

Mann Layne, C., Hohenshil, T., \& Singh, K. (2004). The relationship of occupational stress, psychosocial strain and coping resources to the turnover intentions of rehabilitation counselors. Rehabilitation Counseling Bulletin, $48,19-30$

Martín García, J., Luceño Moreno, L., Jaén Díaz, M., \& Rubio Valdehita, S. (2007). Relación entre factores psicosociales adversos, evaluados a través del cuestionario Decore y salud laboral deficiente. Psicothema, 19, 95-101.

Martínez Selva, J. (2004). Estrés laboral. Guía para empresarios y empleados. Madrid: Prentice Hall.

McClenahan, C., Giles, M., \& Mallett, J. (2007). The importance of context specifity in work stress research: a test of the demand-control-support model in academics. Work \& Stress, 21, 85-95.

McGillis Hall, L., \& Doran, D. (2007). Nurses' perceptions of hospital work environments. Journal of Nursing Management, 15, 264- 273.

McGowan, J., Gardner, D., \& Fletcher, R. (2006). Positive and negative affective outcomes of occupational stress. New Zeeland Journal of Psychology, 35, 92-98.

McNeely, E. (2005). The consequences of job stress for nurses' health: time for a check-up. Nursing Outlook, 53, 291-299.

Montanholi, L. L., Tavares, D. M. S., \& Oliveira, G. R. (2006). Estresse: fatores do risco no trabalho do enfermeiro hospitalar. Revista Brasileira de Enfermagem, 59(6), 661-665.

Moraes, C., Hasselmann, M., \& Reichenheim, M. (2002). Adaptação trascultural dos instrumentos. Cadernos do Saúde Pública, 18, 163-176.

Muhonen, T., \& Torkelson, E. (2004). Work locus of control and its relationship to health and job satisfaction from a gender perspective. Stress and Health, 20, 21-28.

Nacpal, A., \& Shell, B. (1992). Subjective Well-being Inventory. Nueva Delhi: OMS.

Nascimento Ramalho, M., \& Fantini Nogueira-Martins, M. C. (2007). Vivencias de profissionais de saúde da área de oncologia pediátrica. Psicología em Estudo, 12, 123-132.

Naswall, K., Sverke, M., \& Hellgren, J. (2005). The moderating role of personality characteristics on the relationship between job insecurity and strain. Work
\& Stress, 19, 37-49.

Nylenna, M., Gulbrandsen, P., Forde, R., \& Aasland, O. (2005). Unhappy doctors? A longitudinal study of life and job satisfaction among Norwegian doctors 1994-2002. BioMedCentral Health Services Research, 5(44). Extraído en el 23 Marzo de 2006, de http://www.biomedcentral.com/1472-6963/5/44.

Oginska-Bulik, N. (2006). Occupational stress and its consequences in healthcare professionals: the role of type D personality. International Journal of Occupational Medicine and Environmental Health, 19, 113-122.

Omar, A. (2006). Justicia organizacional, individualismo-colectivismo y estrés laboral. Psicología y Salud, 16, 207-217.

Paris, L. (2007). Estrés laboral asistencial, recursos de afrontamiento y satisfacción laboral en trabajadores de la salud de la ciudad de Rosario. Tesis de Doctorado no-publicada, Universidad Nacional de Rosario, Rosario.

Rondeau, K., \& Francescutti, L. (2005). Emergency department overcrowding: the impact of resource scarcity on physician job satisfaction. Journal of Health Care Management, 50, 327-340.

Salmond, S., \& Ropis, P. (2005). Job stress and general well-being: a comparative study of medical-surgical and home care nurses. Medical Surgical Nursing, 14, 301-309.

Shanafelt, T., Novotny, P., Johnson, M., Zhao, X., Steensma, D., Lacy, M., Rubin, J., \& Sloan, J. (2005). The well-being and personal wellness promotion strategies of medical oncologists in the North Central Cancer Treatment Group. Oncology, 68, 23-32.

Shouksmith, G. (1990). A construct validation of a scale for measuring work motivation. New Zealand Journal of Psychology, 18, 76-81.

Skytt, B., Ljunggren, B., \& Carlsson, M. (2007). Reasons to leave: the motives of first-line nurse managers'to leaving their posts. Journal of Nursing Management, 15, 294-302.

Tabak, N., \& Koprak, O. (2007). Relationship between how nurses resolve their conflicts with doctors, their stress and job satisfaction. Journal of Nursing Management, 15, 321-331.

Taylor, D., Pallant, J., Crook, H., \& Cameron, P. (2004). The psychological health of emergency physicians in Australasia. Emergency Medicine Australasia, 16, 21-27.

Ter Doest, L., \& de Jonge, J. (2006). Testing causal models of job characteristics and employee well-being: a replication study using cross-lagged structural equation modelling. Journal of Occupational and Organizational Psychology, 79, 499-507.

Thomas, M. (2005). Team learning: coping with stress. Work Based Learning in Primary Care, 3, 169-172.

Tugade, M., Fredrickson, B., \& Barret, L. (2004). Psychological resilience and positive emotional granularity: examining the benefits of positive emotions on coping and health. Journal of Personality, 72, 1161-1189.

Wainer, J. (2004). Work of female rural doctors. Australian Journal of Rural Health, 12, 49-53.

Wilson, C. (2006). Why stay in nursing? Nursing Management, 12, 24-32.

Zacché Avellar, L., Iglesias, A., \& Fernández Valverde, P. (2007). Sofrimento psíquico em trabalhadores de enfermagem de uma unidade de oncologia. Psicología em Estudo, 12, 475-481.

Zaldúa, G., \& Lodieu, M. (2000). El burnout. La salud de los trabajadores de la salud. Revista del Instituto de Investigaciones de la Facultad de Psicología / UBA, 5(1), 151-169.

Zambrano Plata, G. (2006). Estresores en las unidades de cuidado intensivo. Aquichán, 6, 156-169. 
1. Este trabajo es parte de la Tesis Doctoral en Psicología de la primera autora, realizada bajo la orientación de la segunda.

Laura Paris, Doctora en Psicología por la Universidad Nacional de Rosario (Argentina), es docente de la Facultad de Derecho y Ciencias Sociales del Rosario, de la Universidad Católica Argentina, Argentina. Dirección postal: Sarratea 440 bis, 2000 Rosario, Santa Fe, Argentina. Teléfono: (0341) 155630102. E-mail: laurap_408@yahoo.com.ar Alicia Omar, Doctora en Psicología por la Universidad Nacional de San Luis (Argentina), es investigadora en el Consejo Nacional de Investigaciones Científicas y Técnicas (CONICET), Argentina. E-mail: agomar@ arnet.com.ar 